\title{
Design a Novel Microstrip Rectangle Patch Antenna Utilized in Telemedicine Applications
}

Hamidreza Shirzadfar* and Parisa Shirvani

Department of Biomedical Engineering, Sepahan University, Isfahan, Iran

\section{Editorial}

Use of telecommunication in health care and telemedicine started from the civil war era to the present. The information and communication technologies (ICTs) provide an ability in medical service to overcome geographical barriers and help to provide better health care services. Telemedicine, a term coined in the 1970s which means "healing at a distance" [1], is the use of medical information and telecommunication to improve, maintain, or aid to patients' health status from a distance. The rural and underserved communities can benefit to get more health services to save lives, in order to there are not enough health facilities in their home area. Telemedicine application has a vital role to improve future disaster medicine. As telecommunication technology advances such as computers, broadband internet, and videoconferencing equipment, the main role of telemedicine in health and healing patients will be bolded [2]. We should point out that telemedicine will develop if telecommunication technology progress.

In the field of telecommunication, antennas have crucial role in telemedicine because of antennas send necessary information from a distance at real time. So, design an antenna with great characteristic can be an extremely valuable step to growth telemedicine. Consequently, it is important to focus on the design of antenna utilized in telemedicine.

The many forms of microstrip patch antenna is used in telemedicine such as square, rectangle, circular and triangle $[3,4]$. The design of microstrip patch antenna requested to know and calculate the vital parameters like dielectric constant ( $\varepsilon r$ ), resonant frequency (fr) and dielectric substrate's height (h) [5]. By using noted parameters, it is more appropriate to calculate the patch antenna's dimensions. The dielectric constant is usually in the range of $(2.2 \leq \varepsilon r \leq 12)$.

The utilized and popular frequency of telemedicine applications is equal to $2.45 \mathrm{GHz}$. This frequency is including in ultra-high frequency (UHF) band and the reasons to use this range of frequency are [6].

1. The UHF band is free of charge in the whole of the word.

2. There is no frequency interference at $2.45 \mathrm{GHz}$.

3. The mentioned frequency is applied in Wi-Fi telecommunication.

The one kind of microstrip patch antenna is microstrip yagi patch antenna. This model of antenna is simulated by several researchers at high frequency structure simulation (HFSS). The results which obtained by numerous simulation methods improved return loss, low front to back ratio $[7,8]$. Another design of novel antenna has been presented that has fractal shape at the edges and a square shape slot at the center of the radiating element of a patch antenna. Finlay, this antenna has size reduction and better resonating characteristic [9]. A new configuration of micro strip rectangle patch antenna (MRPA) on different thickness substrate for telemedicine applications are proposed in medicine domain which consists of two parallel conducting layers separated by a single dielectric substrate. The dielectric substrate layer thickness has important parameter to calculate and simulate of microstrip patch antenna. As presented in Table 1, we achieved various results according the different thickness [10]. As presented in

\begin{tabular}{|c|c|}
\hline Thickness $(\mathbf{m m})$ & Gain $(\mathbf{d B})$ \\
\hline 3.175 & 8.1473 \\
\hline 4 & 8.3574 \\
\hline 4.808 & $7.7545 \mathrm{~dB}$ \\
\hline
\end{tabular}

Table 1: The simulation results obtained by HFSS simulator for different thicknesses of dielectric layer.

Table 1, the offered patch antenna has high gain which is significant for telemedicine.

\section{References}

1. Strehle EM, Shabde N (2006) One hundred years of telemedicine: does this new technology have a place in paediatrics. Arch Dis Child 12: 956-959.

2. Pattichis CS (2002) Wireless telemedicine systems: an overview. IEEE Antennas and Propagation Magazine 44: 143-153.

3. Pozar DM, Schaubert DH (1995) Microstrip antennas: the analysis and design of microstrip antennas and arrays. John Wiley \& Sons.

4. Pozar DM (1992) Microstrip antennas. Proceedings of the IEEE 80: 79-91

5. Balanis CA (2016) Antenna theory: analysis and design. John Wiley \& Sons.

6. Wootton R, Craig J, Patterson V (2006) Introduction to telemedicine. London: Royal Society of Medicine Press.

7. Kaneda N (2002) A broadband planar quasi-Yagi antenna. IEEE Transactions on Antennas and Propagation 50: 1158-1160.

8. Xiaohua Yi, Cho C, Cook B, Wang Y, Tentzeris MM, et al. (2014) A Slotted Patch Antenna for Wireless Strain Sensing." Structures Congress.

9. Nathan C (2002) Fractal antenna ground counterpoise, ground planes, and loading elements and microstrip patch antennas with fractal structure. US Patent 6: 476-766.

10. Parisa S, Hamidreza S (2016) Design a New Configuration of Micro Strip Rectangle Patch Antenna on Different Thickness Substrate for Telemedicine Applications. J Nano Electronic Phys.

*Corresponding author: Hamidreza Shirzadfar, Department of Biomedical Engineering, Sepahan University, Isfahan, Iran, Tel: +983136530125; Fax: +983136530245; E-mail: h.shirzadfar@sepahan.ac.ir

Received September 20, 2016; Accepted September 22, 2016; Published September 26, 2016

Citation: Shirzadfar H, Shirvani P (2016) Design a Novel Microstrip Rectangle Patch Antenna Utilized in Telemedicine Applications. J Bioanal Biomed 8: e145. doi: 10.4172/1948-593X.1000e145

Copyright: (c) 2016 Shirzadfar H, et al. This is an open-access article distributed under the terms of the Creative Commons Attribution License, which permits unrestricted use, distribution, and reproduction in any medium, provided the original author and source are credited. 